\title{
Study on the Joint Toxicity of Multi-component Mixtures of Quaternary Ammonium Compounds
}

\author{
Y. Jin*, L.Y. Mo*(**)(***)†, L.T. Qin*(**)(***) and J.F. Dai*(**)(***) \\ *College of Environmental Science and Engineering, Guilin University of Technology, Guilin 541004, China \\ **Technical Innovation Center of Mine Geological Environmental Restoration Engineering in Southern Karst Area, \\ MNR, Nanning 530023, China \\ ***Guangxi Collaborative Innovation Center for Water Pollution Control and Water Safety in Karst Area, Guilin \\ University of Technology, Guilin 541004, China \\ $\dagger$ Corresponding author: Ling-Yun Mo; molingyun123@126.com
}

Nat. Env. \& Poll. Tech.

Website: www.neptjournal.com

Received: 20-11-2020

Revised: $02-03-2021$

Accepted: 24-04-2021

Key Words:

QACs

Multicomponent mixtures

Joint effects

Non-equitoxic ratio

\begin{abstract}
Pollutants generally exist as mixtures in the environment. Their cumulative toxicity and toxicity interactions are potential risks. Therefore, this study aimed to examine the variation of joint toxicity of a multi-component mixture system, which consisted of six common quaternary ammonium salt surfactants in the environment, on Vibrio qinghaiensis sp.-Q67 (Q67). Vibrio qinghaiensis sp. -Q67 (Vqin-Q67) is a freshwater luminescent bacterium that continuously emits blue-green light $(485 \mathrm{~nm})$. The bacterium has been widely used for detecting toxic contaminants. In the mixture system, the luminescent toxicity of each component of the mixture to Q67 was determined by the microplate toxicity analysis method, and the toxicity interaction of the mixture was determined by the toxicity unit method (TU). The combined toxicity of the mixture system was investigated from four aspects, including the number of components, key components, concentration (toxicity) ratio, and exposure time. The results showed that the combined toxic effect of the same mixture system tends to be an additive effect with the increase of the number of components. The combined toxicity of the mixture system was close to that of the key components. Antagonism was presented in the equal toxicity mixture, while synergism was presented in the non-equal toxicity mixture. The combined toxic effect of the multi-component mixture system was not only related to the concentration of the pollutant but also related to the exposure time of the pollutant.
\end{abstract}

\section{INTRODUCTION}

Quaternary ammonium compounds (QACs) are widely used as surfactants and disinfectants (Luo et al. 2020). It has a global output of more than 500000 tons.year $^{-1}$ and has been listed in the list of high-yield chemicals by the Organization for Economic Cooperation and Development (OECD 2004). They are widely used mainly as disinfectants, detergents, preservatives, and fabric softeners (Ruan et al. 2014, Oh et al. 2014, Zhang et al. 2015). Although surfactants have been widely used, most of the products have not been properly treated and discharged into the water body, resulting in serious water environmental pollution (Brycki et al. 2014, Jardak et al. 2016, Ostman et al. 2017). Surfactants are often detected in surface water at the concentration of ug. $L^{-1}$ (Ferrer \& Furlong 2001, Olkowska et al. 2013). Surfactants may threaten the aquatic ecosystems when they reach a certain concentration in the water body (Rosety et al. 2001, Kobuke 2002).

Contaminants are often present in the form of mixtures (Cipullo et al. 2019, Perez \& and Hoang 2017). QACs generally exist as mixtures in the environment (Kwon et al. 2019, Ruan et al. 2014, Zhang et al. 2015). Warne \& Hawker (1995) studied a new hypothesis, the funnel hypothesis, which was derived to explain the variation in toxicity of equitoxic multicomponent mixtures of nonspecific toxicants (narcotics). Results showed that as the number of components in a mixture increases, the range of deviation from toxic additivity decreases. In addition to the number of components and the nature of the compound, the toxicity of the single component also has a large change in the combined toxicity effect ( $\mathrm{Li}$ et al. 2017). Mixture systems of different components can be considered as one space, and mixtures of different mixture ratios are points dispersed in space (Liu et al. 2016a, Liu et al. 2016b). Therefore, the closer mixtures in this space have similar toxicity because their mixing ratios are similar to the concentration levels (Qu et al. 2019), and most studies have shown that component concentration ratio changes also have an effect on the combined toxicity of the mixture (Feng et al. 2017, Xu et al. 2018, Baek et al. 2019). In addition, considerable attention has been given to 
the impact of the exposure period on pollutant toxicities. In the standard toxicity test of algae, bacteria, and large cockroaches, prolonging the exposure time will increase the toxicity of poisons (Vannini et al. 2018, Mo et al. 2020a, Mo et al. 2020b, Hatano \& Shoji 2010). Therefore, it can be found that the number of components in a mixture, the component properties (key components), the concentration (toxicity ratio), and the exposure time are the four main factors affecting the combined toxicity effect of the mixture.

However, most of the current research concentrated on the compound system of equal toxicity ratio ( $\mathrm{Li}$ et al. 2017, Feng et al. 2017, Xu et al. 2018). There is still a lack of a systematic and comprehensive study on the variation law of the combined toxicity effect of the pollutant with complex toxic mechanism and non-toxic ratio mixture system. Therefore, in this study, the quaternary ammonium salt surfactant was used as the research object to determine the combined toxicity effect of a series of mixtures, including binary, ternary, quaternary, five-component mixture, etc.) components. The main factors affecting the toxic effects of the mixture were further revealed. The variation of the toxic effects of the multi-component mixtures was investigated from four aspects: the number of components, the key components, the toxicity (concentration) ratio, and the exposure time.

\section{MATERIALS AND METHODS}

\section{Main Reagent}

The physicochemical properties of the six quaternary ammonium surfactants are shown in Table 1. The stock solution was prepared using Milli-Q ultrapure water and stored in a refrigerator at $4{ }^{\circ} \mathrm{C}$.

\section{Toxicity test and Mixture Design}

The freeze-dried Vibrio qinghaiensis sp.-Q67 (Q67) was purchased from Beijing Hamamatsu Corp., Ltd. (Beijing, China). The formulations of liquid medium, concentrated medium, and solid medium were described in the literature (Yu et al. 2014). Toxicity was determined by microplate toxicity analysis (Yuan et al. 2011).

To investigate the effects of the number of components on the combined toxicity effect, we designed a series of mixture systems with equivalent effect concentration ratios at multiple equal toxicity concentration ratios according to the toxicity data of single QACs compounds. The toxicities of the mixtures were determined by using Q67 as the indicator organism, and the combined toxicities of the mixtures were calculated by using the $T U$ method.

As early as 1965, Sprague \& Ramsay (1965) proposed the concept of toxicity unit (TU) to study the effects of $\mathrm{Cu}-$ $\mathrm{Zn}$ interaction on the growth and development of Atlantic salmon larvae. In 1975, Anderson and Webbe (1975) revised, improved, and developed this concept.

$$
\mathrm{TU}_{\text {sum }}=\frac{\mathrm{C}_{\mathrm{A}}}{\mathrm{EC}_{50-\mathrm{A}}}+\frac{\mathrm{C}_{\mathrm{B}}}{\mathrm{EC}_{50-\mathrm{B}}}+\ldots+\frac{\mathrm{C}_{\mathrm{i}}}{\mathrm{EC}_{50-\mathrm{i}}}
$$

$C_{\mathrm{A}}$ and $C_{\mathrm{B}}$ are the concentrations of the component $\mathrm{A}$ and $\mathrm{B}$ in the mixture system when the mixture is $50 \%$ inhibited, and $E C_{50-\mathrm{A}}$ and $E C_{50-\mathrm{B}}$ are the concentrations when a single compound is $50 \%$ inhibited alone. $T U_{\text {sum }}=1.00 \pm 0.20$ indicates that the combined toxic effect is added, $T U_{\text {sum }}<0.80$ indicates synergism, and $T U_{\text {sum }}>1.20$ indicates antagonism (Broderius et al. 1995).

\section{RESULTS AND DISCUSSION}

\section{Variation of Toxicity Interaction of QACs with the Number of Components}

Before determining the toxicity of the mixture, the acute toxicity of a single compound to Q67 was first determined and we obtained the $\mathrm{EC}_{50}$ of a single compound, which was shown in Table 1:

The toxicity of the mixture was measured according to the toxicity assay method, the combined toxicity effect was

Table 1: Basic Information and $\mathrm{pEC}_{50}$ of Single Compound.

\begin{tabular}{|llllllll|}
\hline No. & Abbr $^{\mathrm{a}}$ & Compound & CAS & MW & Purity $(\%)^{\mathrm{b}}$ & Sources $^{\mathrm{c}}$ & $\mathrm{pEC}_{50}{ }^{\mathrm{d}}$ \\
\hline 1 & $\mathrm{BLB}$ & $\mathrm{C}_{21} \mathrm{H}_{38} \mathrm{BrN}$ & $7281-04-1$ & 384.44 & AR & TRC & 5.130 \\
2 & $\mathrm{TLB}$ & $\left(\mathrm{CH}_{3} \mathrm{CH}_{2}\right)_{4} \mathrm{NBr}$ & $71-91-0$ & 210.16 & AR & TRC & 0.876 \\
3 & $\mathrm{BLC}$ & $\mathrm{C}_{13} \mathrm{H}_{22} \mathrm{ClN}$ & $56-37-1$ & 227.77 & AR & TRC & 2.251 \\
4 & $\mathrm{CTE}$ & $\mathrm{C}_{17} \mathrm{H}_{38} \mathrm{BrN}$ & $1119-97-7$ & 336.39 & AR & TRC & 4.885 \\
5 & TAC & $\mathrm{C}_{16} \mathrm{H}_{36} \mathrm{ClN}$ & $214-195-7$ & 277.92 & AR & TRC & 4.795 \\
6 & DTC & $\mathrm{C}_{15} \mathrm{H}_{34} \mathrm{~N} \cdot \mathrm{Cl}$ & $112-00-5$ & 263.89 & AR & TRC & 2.724 \\
\hline
\end{tabular}

a Abbreviation for a single compound name; b: Analytical purity.

c Truth and Reconciliation Commission of Canada; d: Negative logarithm of 50\% effect concentration. 
calculated using $\mathrm{TU}_{\text {sum. }}$. The results are shown in Table 2 and Fig. 1.

The binary equivalent concentration ratio $\left(\mathrm{EC}_{50}\right)$ of $\mathrm{BLB}$ TLB was used as the initial mixture, and different QACs were added into the binary mixture according to the equivalent concentration ratio $\left(\mathrm{EC}_{50}\right)$, in turn, to form a series of multi-component equivalent concentration ratio mixtures (Table 2). Since it was difficult to determine the single toxicity of 22 compounds and the combined toxicity of their mixtures simultaneously, the 22 multi-component mixtures used in this study were all 22-component mixtures. As indicated in Table 1 and Fig. 1a, the combined toxic effect of the multivariate isotopic concentration ratio changed from antagonistic to additive effect with the consecutive addition of the QACs chemical for that binary isotopic system of BLB-TLB as the initial system. Fig. 1b was a histogram of the mixture, the original quaternary mixing system was a multi-hybrid system in which toxic effects were antagonistic. The combined toxic effects of a mixing system tend to have an additive effect as the number of components in the combination increases.

The above results showed that the mixture system had a similar trend when the mixture was mixed with an equal effect concentration ratio for the same system of pollutants. The antagonistic effect increased first and then decreased gradually to concentration addition with the increase of component. For example, as shown in Fig. 1a, the apparent joint toxicity of the six-membered mixture was antagonism $\left(\mathrm{TU}_{\text {sum }}=2.53 \pm 0.05\right)$ and the joint toxicity of the 22-membered mixture was additive $\left(\mathrm{TU}_{\text {sum }}=0.81 \pm 0.05\right)$, which indicated that the number of components mixture system had an effect
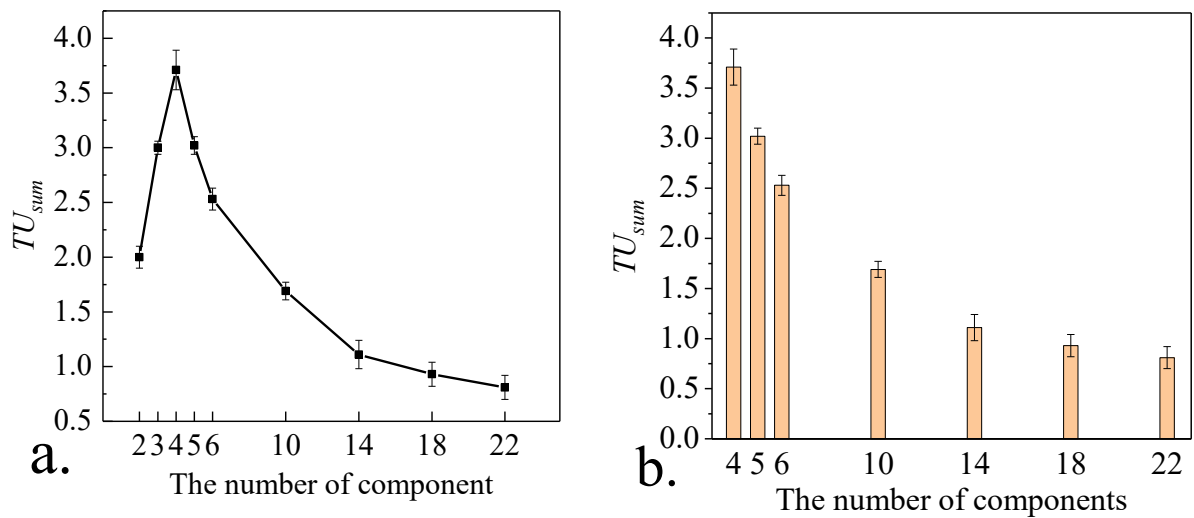

Fig. 1: Effect of Component Number on the Toxicity Effect of Multi-Equivalent Mixture System with Equivalent Effect Concentration Ratio (EC ${ }_{50}$ ). ( $a$ is $\mathrm{CRC}$ of the mixture; $\mathrm{b}$ is a histogram of the mixture).

Table 2: Composition of compound pollutants and determination results.

\begin{tabular}{|c|c|c|c|c|c|c|c|c|}
\hline Number of components & 1 & 2 & 3 & 4 & 5 & 6 & $\mathrm{TU}_{\text {sum }}$ & $95 \% \mathrm{CI}^{\mathrm{e}}$ \\
\hline 2 & BLB & TLB & & & & & 2 & $1.95 \sim 2.05$ \\
\hline 3 & BLB & TLB & BLC & & & & 3 & $2.97 \sim 3.03$ \\
\hline 4 & BLB & TLB & BLC & CTE & & & 3.71 & $3.62 \sim 3.80$ \\
\hline 5 & BLB & TLB & BLC & CTE & DTC & & 3.02 & $2.98 \sim 3.06$ \\
\hline 6 & BLB & TLB & BLC & CTE & DTC & TAC & 2.53 & $2.48 \sim 2.58$ \\
\hline $10 \mathrm{a}$ & BLB & TLB & BLC & CTE & DTC & TAC & 1.69 & $1.64 \sim 1.72$ \\
\hline $14 b$ & BLB & TLB & BLC & CTE & DTC & TAC & 1.11 & $1.08 \sim 1.21$ \\
\hline $18 \mathrm{c}$ & BLB & TLB & BLC & CTE & DTC & TAC & 0.93 & $0.87 \sim 0.98$ \\
\hline $22 \mathrm{~d}$ & BLB & TLB & BLC & CTE & DTC & TAC & 0.81 & $0.76 \sim 0.86$ \\
\hline
\end{tabular}

a Includes single $\mathrm{EC}_{50} \mathrm{BLB}+\mathrm{TLB}$ and four 2-fold $\mathrm{EC}_{50} \mathrm{BLC}+\mathrm{CTE}+\mathrm{DTC}+\mathrm{TAC}(2+4 \times 2=10)$;

$\mathrm{b}$ Includes single $\mathrm{EC}_{50} \mathrm{BLB}+\mathrm{TLB}$ and four 3 -fold $\mathrm{EC}_{50} \mathrm{BLC}+\mathrm{CTE}+\mathrm{DTC}+\mathrm{TAC}(2+4 \times 3=14)$;

c Includes single $\mathrm{EC}_{50} \mathrm{BLB}+\mathrm{TLB}$ and four 4 -fold $\mathrm{EC}_{50} \mathrm{BLC}+\mathrm{CTE}+\mathrm{DTC}+\mathrm{TAC}(2+4 \times 4=18)$;

$\mathrm{d}$ Includes single $\mathrm{EC}_{50} \mathrm{BLB}+\mathrm{TLB}$ and four 5 -fold $\mathrm{EC}_{50} \mathrm{BLC}+\mathrm{CTE}+\mathrm{DTC}+\mathrm{TAC}(2+4 \times 5=22)$.

e $95 \%$ Confidence interval. (the same below) 
on the joint toxicity of the mixture system. Therefore, these components can be considered as the key components to determine the joint toxic effects of multi-component mixtures.

\section{Variation of Toxicity Interaction of QACs with Key Components}

Fig. 2 showed the effects of key components of the isotope ratio mixture on the TU values. In Fig. 2a, the combined toxic effects of adding 2-fold TAC and 2-fold DTC became stronger. The experimental results of Fig. 2(b-d) further validated the joint toxic effects of binary, ternary, and quaternary mixtures with increasing composition.

The experimental results of Fig. 2(b-d) further validated the joint toxic effects of binary, ternary, and quaternary mixtures with increasing composition. For the binary mixture of BLB and TLB (Fig.2b), the antagonistic effect was weakened by the addition of BLC, because the combined effect of BLB or TLB in the BLC and the original binary system is stronger than that of BLB and TLB. For the binary mixture of BLB and BLC, the antagonistic effect was weakened by adding TLB, because TLB and BLB could produce a strong antagonistic effect, which was stronger than the concentration addition effect of the original binary mixture of BLB and BLC. As shown in Fig. 2c and Fig. 2d, except for the BLB+BLC+CTE, isovolumetric mixing in Fig. 2c presented a concentration addition effect, and the BLB+BLC+CTE+DTC isovolumet- ric mixing in Fig. 2d induced a synergistic effect, which was similar to the other mixtures in Fig. $2 a$.

Fig. 2a showed that the results of this study were not consistent with the funnel hypothesis proposed by Warne and Hawker (2010). The results showed that not only the number of components in the mixture system but also the properties of components (especially the key components) have an effect on the joint toxicity. In these multi-component mixtures, the components that produce the strongest synergistic effects in binary mixtures could be considered as the key components.

It could be seen from Table 3 and Fig. 2 that the combined toxic effects of the mixture system could be enhanced when the newly added components and one of the original components in the system had strong antagonistic effects. The components with strong combined effects could be regarded as the key components to determine the joint toxic effects of multi-component mixtures. The contribution of these key components to the joint toxic effects of multicomponent mixtures was greater than that of other components. The results were showed that the joint toxic effects of multi-component mixtures were close to the joint toxic effects of the key components. In other words, the key components that determine the combined toxic effect of multi-component mixed systems can be determined by comparing the toxic effects of a series of mixtures.

Table 3: Composition of compound pollutants and determination results.

\begin{tabular}{|c|c|c|c|c|c|c|}
\hline Mixture & Volume ratio & 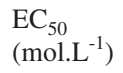 & $\begin{array}{l}-\operatorname{lgEC} \mathrm{C}_{50} \\
\left(\mathrm{~mol}^{-L^{-1}}\right)\end{array}$ & $95 \% \mathrm{CI}$ & $\mathrm{TU}_{\text {sum }}$ & Toxic interaction \\
\hline $\mathrm{BLB}+\mathrm{TLB}$ & $1: 1$ & $1.22 \mathrm{E}-01$ & 0.91 & $0.88 \sim 0.95$ & 1.37 & ANT \\
\hline \multirow[t]{2}{*}{$\mathrm{BLB}+\mathrm{TLB}+\mathrm{BLC}$} & $1: 1: 1$ & $8.61 \mathrm{E}-02$ & 1.06 & $1.00 \sim 1.13$ & 1.27 & Slight ANT \\
\hline & $2: 1: 1$ & $7.53 \mathrm{E}-02$ & 1.12 & $1.08 \sim 1.17$ & 1.39 & ANT \\
\hline \multirow[t]{2}{*}{$\mathrm{BLB}+\mathrm{TLB}+\mathrm{BLC}+\mathrm{CTE}$} & $1: 1: 1: 1$ & $1.07 \mathrm{E}-01$ & 0.97 & $0.89 \sim 1.06$ & 1.96 & ANT \\
\hline & $4: 1: 1: 1$ & $6.28 \mathrm{E}-02$ & 1.20 & $1.11 \sim 1.30$ & 1.85 & ANT \\
\hline $\mathrm{BLB}+\mathrm{TLB}+\mathrm{BLC}+\mathrm{CTE}+\mathrm{DTC}$ & $1: 1: 1: 1: 1$ & 7.27E-02 & 1.14 & $1.08 \sim 1.19$ & 1.61 & ANT \\
\hline $\mathrm{BLB}+\mathrm{TLB}+\mathrm{BLC}+\mathrm{CTE}+\mathrm{DTC}+\mathrm{TAC}$ & $1: 1: 1: 1: 1: 1$ & $2.22 \mathrm{E}-02$ & 1.65 & $1.63 \sim 1.67$ & 0.57 & SYN \\
\hline $\mathrm{BLB}+\mathrm{TLB}+\mathrm{BLC}+\mathrm{CTE}+\mathrm{DTC}+2 \mathrm{TAC}$ & $1: 1: 1: 1: 1: 2$ & $5.62 \mathrm{E}-02$ & 1.25 & $1.22 \sim 1.28$ & 0.52 & SYN \\
\hline $\mathrm{BLB}+\mathrm{TLB}+\mathrm{BLC}+\mathrm{CTE}+2 \mathrm{DTC}+2 \mathrm{TAC}$ & $1: 1: 1: 1: 2: 2:$ & $5.67 \mathrm{E}-02$ & 1.25 & $1.19 \sim 1.30$ & 0.48 & SYN \\
\hline $\mathrm{BLB}+\mathrm{BLC}$ & $1: 1$ & $2.92 \mathrm{E}-03$ & 2.53 & $2.50 \sim 2.57$ & 1.04 & ADD \\
\hline $\mathrm{BLB}+\mathrm{BLC}+\mathrm{CTE}$ & $1: 1: 1$ & $1.51 \mathrm{E}-03$ & 2.82 & $2.79 \sim 2.85$ & 0.80 & Slight ADD \\
\hline $\mathrm{BLB}+\mathrm{BLC}+\mathrm{CTE}+\mathrm{DTC}$ & $1: 1: 1: 1$ & $6.10 \mathrm{E}-04$ & 3.21 & $3.17 \sim 3.26$ & 0.43 & SYN \\
\hline $\mathrm{BLB}+\mathrm{TLB}+\mathrm{CTE}$ & $1: 1: 1$ & $9.15 \mathrm{E}-02$ & 1.04 & $1.00 \sim 1.08$ & 1.38 & ANT \\
\hline $\mathrm{BLB}+\mathrm{TLB}+\mathrm{CTE}+\mathrm{DTC}$ & $1: 1: 1: 1$ & 7.77E-02 & 1.11 & $1.05 \sim 1.16$ & 1.46 & ANT \\
\hline $\mathrm{BLB}+\mathrm{TLB}+\mathrm{BLC}+\mathrm{DTC}$ & $1: 1: 1: 1$ & $8.65 \mathrm{E}-02$ & 1.06 & $1.01 \sim 1.12$ & 1.59 & ANT \\
\hline $\mathrm{TLB}+\mathrm{BLC}+\mathrm{CTE}+\mathrm{DTC}$ & $1: 1: 1: 1$ & $9.68 \mathrm{E}-02$ & 1.01 & $0.97 \sim 1.06$ & 1.78 & ANT \\
\hline
\end{tabular}

ANT: Antagonism (the same below); ADD: Addition (the same below); SYN: Synergism (the same below) 


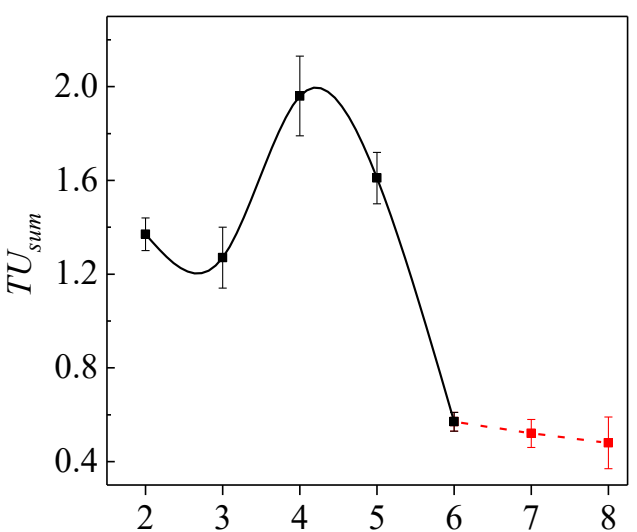

a.

$$
\text { The number of components }
$$

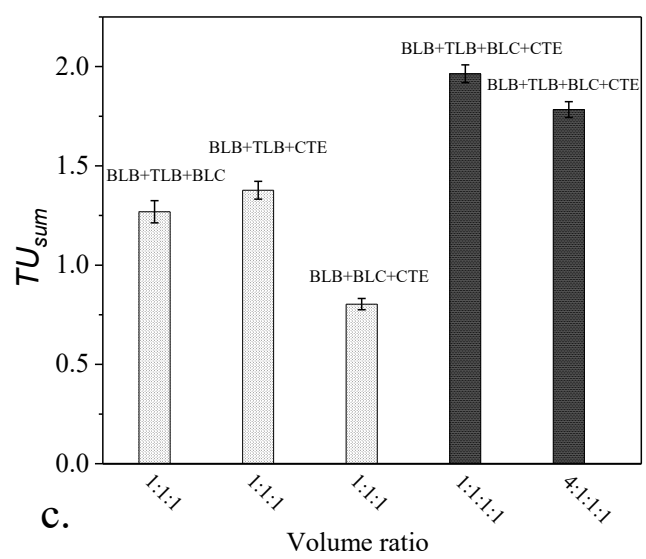

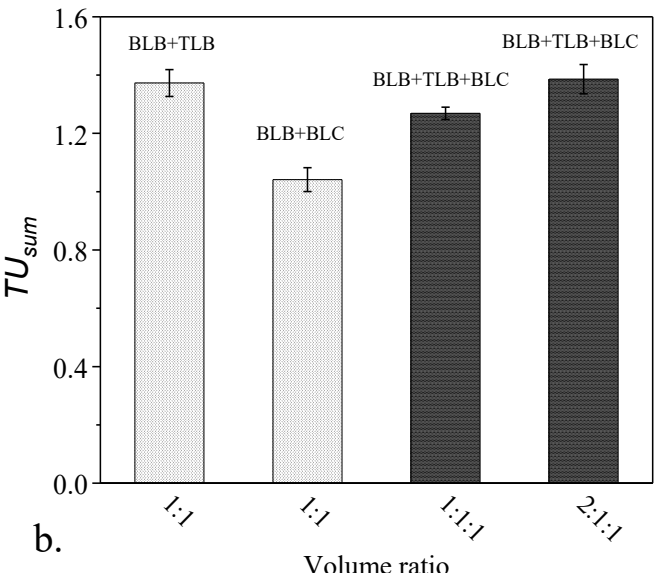

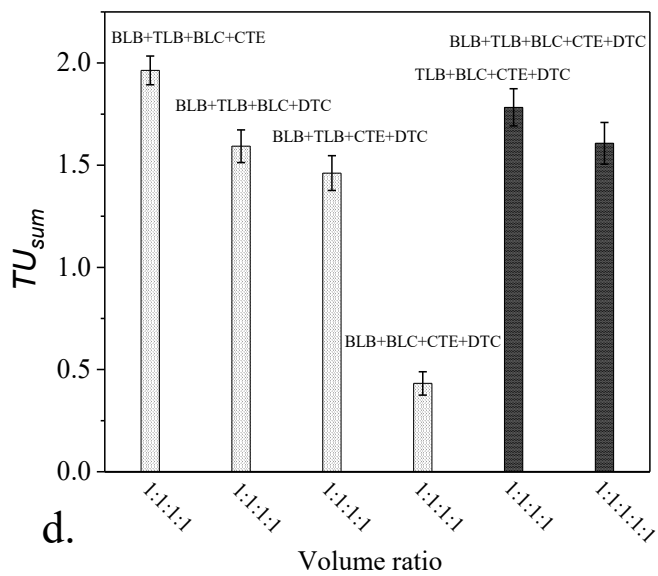

Fig. 2: Effect of key numbers on joint toxicity of mixed systems with multiple equal toxicity ratios.

\section{Variation of Mixed Toxicity Effect of QACs Mixtures with Mixture Concentration}

The combined toxicity effects of binary mixture systems with the equal effect concentration ratio are shown in Fig. 3, Fig. 4, and Table 4.

In Fig. 3a, the toxic effects of BLB+TLB mixture except $\mathrm{EC}_{5}$ mixture were additive effect, and the other equivalent concentration ratios mixture were antagonistic effects. In Fig. 3b, the toxic effects of BLC-CTE mixed with $\mathrm{EC}_{5}$ were additive, and the other equivalent concentration ratios were antagonistic. In Fig. 3c, the equivalent concentration of TAC+DTC was antagonistic at the concentration of $\mathrm{EC}_{5}$, $\mathrm{EC}_{10}, \mathrm{EC}_{20}, \mathrm{EC}_{30}, \mathrm{EC}_{40}$, and $\mathrm{EC}_{50}$, but all the $\mathrm{TU}_{\text {sum }}$ of the mixtures of $\mathrm{EC}_{5}$ were 1.62, and the $\mathrm{TU}_{\text {sum }}$ of the other mixtures was $1.35 \sim 1.42$. In Fig. 3b, BLC+CTE mixture
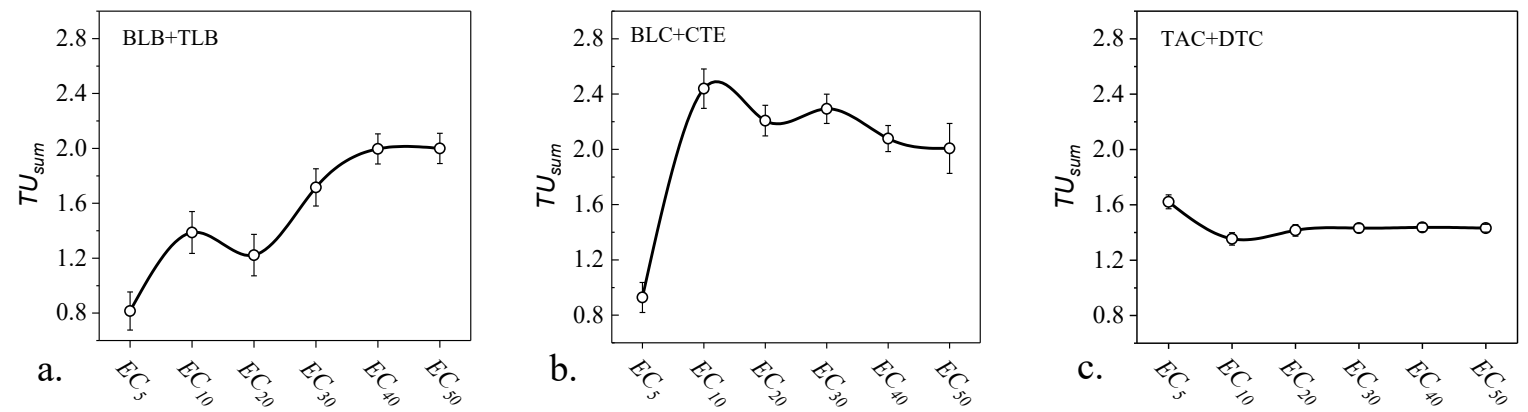

Fig. 3: Effects of mixing with different equivalent concentration ratios on joint toxicity of binary mixtures. 
Table 4: Composition and joint toxicity of binary compounds.

\begin{tabular}{|c|c|c|c|c|c|c|}
\hline Mixture & $\begin{array}{l}\text { Equal effect concentration } \\
\text { ration }\end{array}$ & $\begin{array}{l}E C_{50} \\
(\mathrm{~mol} / \mathrm{L})\end{array}$ & $\begin{array}{l}-\lg E C_{50} \\
(\mathrm{~mol} / \mathrm{L})\end{array}$ & $95 \% \mathrm{CI}$ & $\mathrm{TU}_{\text {sum }}$ & Toxic interaction \\
\hline \multirow[t]{6}{*}{$\mathrm{BLB}+\mathrm{TLB}$} & $\mathrm{EC}_{5}+\mathrm{EC}_{5}$ & $7.50 \mathrm{E}-02$ & 1.12 & $1.10 \sim 1.15$ & 0.81 & Slight ADD \\
\hline & $\mathrm{EC}_{10}+\mathrm{EC}_{10}$ & $1.14 \mathrm{E}-01$ & 0.94 & $0.86 \sim 1.02$ & 1.22 & Slight ANT \\
\hline & $\mathrm{EC}_{20}+\mathrm{EC}_{20}$ & 8.98E-02 & 1.05 & $1.00 \sim 1.09$ & 1.39 & ANT \\
\hline & $\mathrm{EC}_{30}+\mathrm{EC}_{30}$ & $1.19 \mathrm{E}-01$ & 0.93 & $0.85 \sim 1.00$ & 1.72 & ANT \\
\hline & $\mathrm{EC}_{40}+\mathrm{EC}_{40}$ & $1.34 \mathrm{E}-01$ & 0.87 & $0.82 \sim 0.93$ & 1.96 & ANT \\
\hline & $\mathrm{EC}_{50}+\mathrm{EC}_{50}$ & $1.33 \mathrm{E}-01$ & 0.88 & $0.83 \sim 0.93$ & 2.00 & ANT \\
\hline \multirow[t]{6}{*}{ BLC+CTE } & $\mathrm{EC}_{5}+\mathrm{EC}_{5}$ & $2.35 \mathrm{E}-03$ & 2.63 & $2.56 \sim 2.70$ & 0.93 & ADD \\
\hline & $\mathrm{EC}_{10}+\mathrm{EC}_{10}$ & $6.36 \mathrm{E}-03$ & 2.20 & $2.17 \sim 2.23$ & 2.44 & ANT \\
\hline & $\mathrm{EC}_{20}+\mathrm{EC}_{20}$ & $5.94 \mathrm{E}-03$ & 2.23 & $2.18 \sim 2.27$ & 2.21 & ANT \\
\hline & $\mathrm{EC}_{30}+\mathrm{EC}_{30}$ & $6.28 \mathrm{E}-03$ & 2.20 & $2.17 \sim 2.24$ & 2.29 & ANT \\
\hline & $\mathrm{EC}_{40}+\mathrm{EC}_{40}$ & $5.77 \mathrm{E}-03$ & 2.24 & $2.19 \sim 2.28$ & 2.08 & ANT \\
\hline & $\mathrm{EC}_{50}+\mathrm{EC}_{50}$ & $5.64 \mathrm{E}-03$ & 2.25 & $2.16 \sim 2.34$ & 2.01 & ANT \\
\hline \multirow[t]{6}{*}{ TAC+DTC } & $\mathrm{EC}_{5}+\mathrm{EC}_{5}$ & $2.16 \mathrm{E}-03$ & 2.67 & $2.62 \sim 2.71$ & 1.62 & ANT \\
\hline & $\mathrm{EC}_{10}+\mathrm{EC}_{10}$ & $1.80 \mathrm{E}-03$ & 2.75 & $2.72 \sim 2.77$ & 1.35 & ANT \\
\hline & $\mathrm{EC}_{20}+\mathrm{EC}_{20}$ & $1.87 \mathrm{E}-03$ & 2.73 & $2.67 \sim 2.78$ & 1.42 & ANT \\
\hline & $\mathrm{EC}_{30}+\mathrm{EC}_{30}$ & $1.89 \mathrm{E}-03$ & 2.72 & $2.68 \sim 2.76$ & 1.43 & ANT \\
\hline & $\mathrm{EC}_{40}+\mathrm{EC}_{40}$ & $1.89 \mathrm{E}-03$ & 2.72 & $2.68 \sim 2.77$ & 1.44 & ANT \\
\hline & $\mathrm{EC}_{50}+\mathrm{EC}_{50}$ & $1.88 \mathrm{E}-03$ & 2.73 & $2.65 \sim 2.80$ & 1.45 & ANT \\
\hline
\end{tabular}

ANT: Antagonism (the same below); ADD: Addition (the same below); SYN: Synergism (the same below)

systems exhibited additive or antagonistic effects at equal toxicity ratios. It was clear from TAC+DTC in Fig. 3c that the antagonistic effect was present at an equal toxicity ratio. For these two-two mixed systems, their combined toxicity effects did vary with the toxicity ratios.

Fig. 4 showed six component mixtures of five QACs compounds (BLB, TLB, BLC, CTE, and DTC) as the volume ratio of the compounds changed. Fig. 4 a showed a ternary mixture system composed of BLB, TLB, BLC, CTE, and DTC in the ratio $1: 1: 1,2: 1: 1$, and $4: 1: 1$. It could be seen from the figure that the combined toxicities of the mixture of $\mathrm{BLB}+\mathrm{BLC}+\mathrm{DTC}, \mathrm{BLB}+\mathrm{CTE}+\mathrm{DTC}$, and BLC+CTE+DTC mixtures were synergistic effects, and toxic effects did not change with the volume change of a single component. The combined toxicity of the BLB+BLC+CTE mixture was additive and basically did not change with the volume change of a single component. The combined toxicity of the BLB+TLB+BLC mixture was concentration additive. The mixture toxicities of the BLB+TLB+CTE and TLB+BLC+CTE mixtures were antagonism at ratio 1:1:1 and became additive at ratio 4:1:1 with the change of volume ratio of a single component.
Fig. 4b showed three ternary mixtures of BLB, TLB, BLC, CTE, and DTC in 1:1:1, 1:1:2, and 1:1:4. The toxicity of the BLB+TLB+CTE mixture was antagonism, and $\mathrm{TU}_{\text {sum }}$ slightly changed with the increase of volume ratio of CTE, but it did not cause the change of mixture toxicity. The mixture toxicities of BLB+BLC+DTC and BLB+DTC+CTE were synergistic, and the synergistic effect of BLB+BLC+DTC increased slightly with the increase of DTC volume. Fig. $4 \mathrm{c}$ showed two ternary mixtures composed of BLB, TLB, BLC, and CTE of ratios 1:1:1, 1:2:1, and 1:4:1. The mixture toxicity of the BLB+TLB+BLC mixture was antagonistic. As the volume of TLB increases, the mixture toxicity tends to increase from antagonistic effect to additive effect. The mixture toxicity of the BLB+BLC+CTE mixture was the synergistic effect, and the synergistic effect was the strongest when the volume ratio was $1: 2: 1$. Fig. 4 d showed two ternary mixtures of BLC, CTE, TLB, and DTC in 1:1:1, 1:1:5, and $1: 1: 10$. The toxicity of the TLB+BLC+CTE mixture tended to shift from synergistic to antagonistic as the CTE volume increased. With the increase of DTC volume, the $\mathrm{TU}_{\text {sum }}$ of the BLC+CTE+DTC mixture had no change. Therefore, the key component may be CTE. 

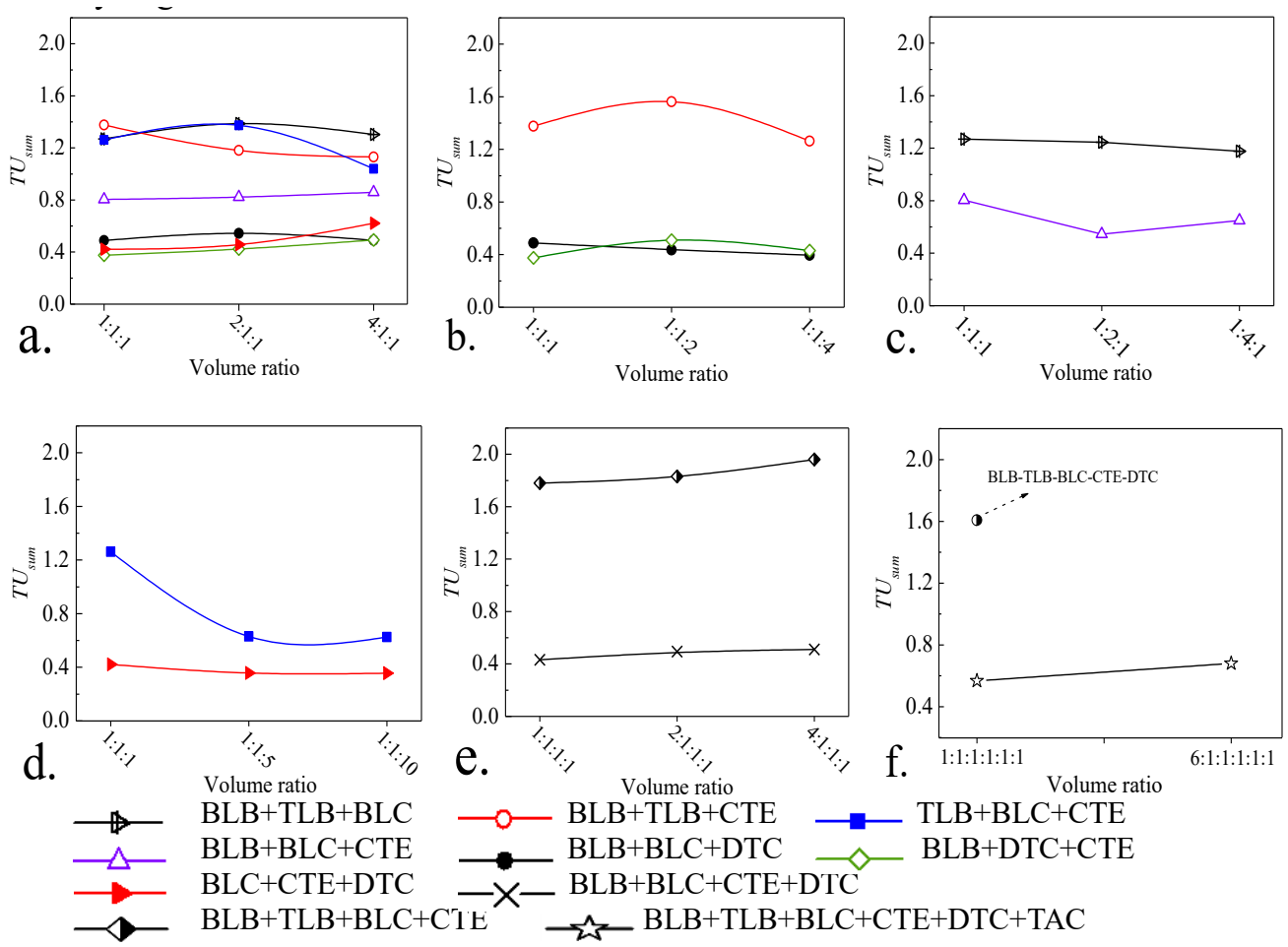

Fig. 4: Ternary and multivariate mixed equal volume ratio.

Fig. 4e shows two quaternary mixtures of BLB, TLB, BLC, CTE, and DTC in $1: 1: 1: 1,2: 1: 1: 1: 1$, and $4: 1: 1: 1$. The mixture toxicity of the BLB+TLB+BLC+CTE mixture was antagonistic, and with the increase of BLB volume, the toxicity $\mathrm{TU}_{\text {sum }}$ had little change. The mixture of BLB+BLC+CTE was added into TLB, which made the mixture toxicity change from additive to antagonistic. The mixture toxicity of BLB+BLC+CTE+DTC was antagonistic. It was the synergistic effect, and $\mathrm{TU}_{\text {sum }}$ slightly increases with the increase of BLB volume. It could be seen that adding DTC to the BLB+BLC+CTE mixture could increase the toxicity of the mixture, while the toxicity of ternary mixture containing DTC did not change with the change of the volume of other components. It could be seen that DTC was the key component in any ternary mixture and quaternary mixture.

Fig. 4f showed six component mixture of ratios $1: 1: 1: 1: 1: 1$ and $6: 1: 1: 1: 1: 1: 1$, and five-component mixture of ratio $1: 1: 1: 1: 1: 1$. It could be seen from the figure that with the increase of BLB volume, the toxicity interaction of the mixture basically had no change. Compared with Fig. 4e, it could be seen that TLB was added to the BLB+BLC+CTE+DTC mixture, and the toxicity interaction of the mixture changed significantly, that was, from synergism to antagonism. It could be seen that TLB may be the key component in the five component mixture. When DTC was added to the BLB+TL-
$\mathrm{B}+\mathrm{BLC}+\mathrm{CTE}$ mixture, the $\mathrm{TU}_{\text {sum }}$ of the mixture decreased slightly, but it was still antagonism. When TAC was added to the mixture of BLB+TLB+BLC+CTE+DTC, the $\mathrm{TU}_{\text {sum }}$ of the mixture interaction decreased significantly, that was, from antagonism to synergism.

It could be seen from Fig. 4 that with the shift of toxicity ratio, i.e. volume ratio, from isotoxicity to non-isotoxicity, its combined toxicity effect changes antagonism-concentration addition-synergy. The results show that the number of fraction items and key components mentioned above, and the toxicity ratio of the components in the mixture system also had an effect on the joint toxicity of the mixed system. Taking the mixture of TLB+ BLC+CTE, BLB+BLC+CTE, and BLB+TLB+BLC+TCE in The toxic effect of a binary mixture containing BLC and CTE is TUsum $=0.932 .44$, increasing the BLB component was a ternary mixture, and the concentration additive effect of BLB and BLC+CTE (TUsum $=0.80 .86$ ) was greater than the existing combined toxicity effect of the binary mixed system and was increased based on the ternary mixture (see Fig. 4). The synergistic effect of DTC and BLC+CTE+BLB $\left(\mathrm{TU}_{\text {sum }}=0.49 \sim 0.51\right)$ was greater than the existing combined toxic effect of the ternary mixed system. The toxic effect was enhanced after increasing DTC. However, for the toxic effect of the binary mixtures containing $\mathrm{BLB}$ and $\mathrm{CTE}\left(\mathrm{TU}_{\text {sum }}=0.8 \sim 2.00\right)$, 
except $\mathrm{EC}_{5}$, which is 0.8 , it was $1.39 \sim 2.00$. The combined toxicity of other mixtures was less than the existing effect of ternary mixtures $(\mathrm{BLB}+\mathrm{CTE}+\mathrm{BLC})\left(\mathrm{TU}_{\text {sum }}=1.27 \sim 1.38\right)$, so the increase of BLC enhanced the combined toxicity effect of the mixed system.

\section{Variation of Mixture Toxicity of QACs with Different Exposure Time}

Fig. 5 showed the change of combined toxic effect of five components $(\mathrm{BLB}+\mathrm{TLB}+\mathrm{BLC}+\mathrm{CTE}+\mathrm{DTC})$ mixture system with exposure time. It was shown that the mixture system with equal effect concentration ratio $\mathrm{EC}_{50}$ and $\mathrm{EC}_{10}$ was antagonistic effects. With the increase of exposure time ( $0.5 \mathrm{~h}, 2 \mathrm{~h}, 4 \mathrm{~h}, 6 \mathrm{~h}, 8 \mathrm{~h}, 10 \mathrm{~h}$, and $12 \mathrm{~h})$, the toxic effect of $\mathrm{EC}_{50}$ mixture systems was gradually weakened. When the mixture was mixed according to $\mathrm{EC}_{10}$, its toxic effect gradually weakened with the increase of exposure time, and it was the weakest when the exposure time was $4 \mathrm{~h}$.

As could be seen from Fig. 5, in the mixture system, it was found that the joint toxic effect of the multiple mixture system was not only related to the dose or concentration of pollutants but also related to the exposure time of pollutants.

\section{Tendency Analysis of Mixed Toxic Effects of QACs}

To further analyze the trend of joint toxic effects of multi-component mixtures, we converted the multi-component mixtures into corresponding fraction numbers based on the toxicity ratios of the multicomponent mixtures. For example, a ternary mixture with a toxicity ratio of $4: 1: 1$ could be considered as a mixture of six components. Then, the relationship between the combined effect of the mixture and the purpose of the fraction could be obtained. As the purpose of the fraction increases, the possibility of antagonistic or synergistic effects increases gradually. However, it should be noted that it was very difficult to obtain a uniform critical number of components for different reaction-type mixtures. This was because the joint toxicity of the mixture was not

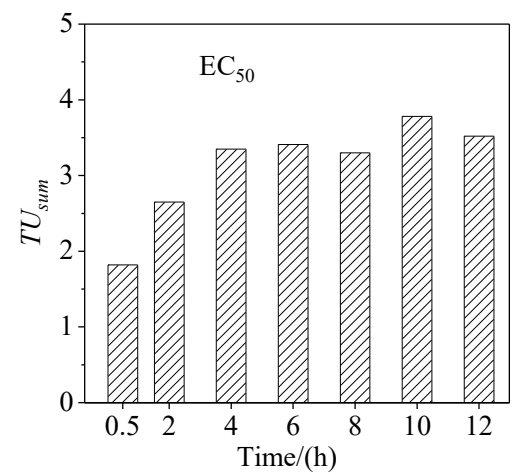

only related to the number of components, but also to the key components, toxicity ratio, and exposure time.

To summarize, those components which can produce strong combined effects were the key components to determine the combined toxic effects of the mixture system. Therefore, we used $\mathrm{TU}_{\min }$-binary to describe quantitatively the contribution of the key components in 3, 4- and 5-component mixtures to the mixture system. In the regions with large values of $\mathrm{TU}_{\mathrm{min}}$-binary and non-isotoxicity ratios, the joint toxic effects of multi-component mixtures tended to antagonistic effects. The joint toxic effects of multicomponent mixtures tend to have synergistic effects in the region where the values of $\mathrm{TU}_{\min }$-binary and non-isotopic ratios were small because the joint toxic effects of multicomponent mixtures in this region were not determined by a single factor, but by a combination of multiple factors.

\section{CONCLUSION}

The results showed that the changes of joint toxic effects were mainly affected by four factors: the number of components, the properties of key components, concentration of components, and the exposure time. In general, the antagonism of the combined effect increased with the increase of the fraction when the newly added components had a strong combined effect with the existing components. On the contrary, if the added components can have a strong combined effect with the existing components, and this effect was much stronger than the toxic effect between the existing components, then the toxic effect of the mixture system would be enhanced with the increase of the new components.

\section{ACKNOWLEDGEMENTS}

The authors are especially grateful to the financial support from the National Natural Science Foundation of China (21667013), National Key Research and Development Program of China (2019YFC0507502), Natural Science Founda-

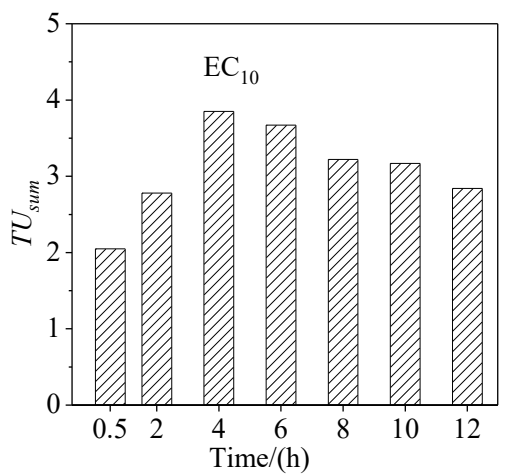

Fig. 5: Effects of a mixture of quintile equivalent concentration ratios on joint toxicity of Q67 in different exposure time. 
tion of Guangxi Province (2018GXNSFAA281156), Guilin Scientific Research and Technology Development Program (20180107-5, 20180101-1 and 20190216-2).

\section{REFERENCES}

Anderson, P.D. and Weber L.J. 1975. The toxicity of aquatic populations of mixtures containing certain heavy metals. In: Proceedings of the International Conference on Heavy Metals in the Environment Canada, 27-31 October 1975, The University of Toronto, Toronto Institute of Environmental Studies, pp. 933-953.

Baek, I.H., Kim, Y., Baik, S. and Kim, J. 2019. Investigation of the synergistic toxicity of binary mixtures of pesticides and pharmaceuticals on Aliivibrio fischeri in major river basins in South Korea. Int. J. Environ. Res. Pub. Health, 16: 148-162.

Broderius, S.J., Kahl, M.D. and Hoglund, M.D. 1995. Use of joint toxic response to define the primary mode of toxic action for diverse industrial organic chemicals. Environ. Toxicol. Chem., 14(9): 1591-1605.

Brycki, B., Walig Orska, M. and Szulc, A. 2014. Nomeric: The biodegradation of monomeric and dimeric alkylammonium surfactants. J. Hazard. Mater., 280: 797-815.

Cipullo, S., Snapir, B., Prpich, G., Campo, P. and Coulon, F. 2019. Prediction of bioavailability and toxicity of complex chemical mixtures through machine learning models. Chemosphere, 215: 388-395.

Feng, L., Liu, S.S., Li, K., Tang, H.X. and Liu H.J. 2017. The time-dependent synergism of the six-component mixtures of substituted phenols, pesticides, and ionic liquids to Caenorhabditis elegans. J. Hazard. Mater., 327: 11-17.

Ferrer, I. and Furlong, E.T. 2001. Identification of alkyl dimethyl benzyl ammonium surfactants in water samples by solid-phase extraction followed by ion trap LC/MS and LC/MS/MS. Environ. Sci. Technol., 35: 2583-2588.

Jardak, K., Drogui, P. and Daghrir, R. 2016. Surfactants in the aquatic and terrestrial environment: occurrence, behavior, and treatment processes. Environ. Sci. Pollut. Res., 23: 3195-3216.

Hatano, A. and Shoji, R. 2010. A new model for predicting the time course toxicity of heavy metals based on the biotic ligand model (BLM). Comp. Biochem. Physiol. Part - C: Toxicol. Pharmacol., 151: 25-32.

Kobuke, Y. 2002. Environmental risk evaluation for anionic surfactants based on the characteristics of the appearance of high concentration in rivers. Water Sci. Technol., 46(11): 263-268.

Kwon, D., Lim, Y.M., Kwon, J.T., Shim, I., Kim, E., Lee, D.H. Yoon, B.I., Kim, P. and Kim, H.M. 2019. Evaluation of pulmonary toxicity of benzalkonium chloride and triethylene glycol mixtures using in vitro and in vivo systems. Environ. Toxicol., 34(5): 561-272.

Li, K., Liu, S.S. and Qu, R. 2017. Application of the combination index in the assessment of combined toxicity of environmental mixture. Asian J. Ecotoxicol., 12: 62-71.

Liu, S.S., Li, K., Li, T. and Qu, R. 2016a. Comments on the synergistic toxicity of the multi chemical mixtures: Implications for risk assessment in the terrestrial environment. Environ. Int., 94: 396-398.

Liu, S.S., Xiao, Q.F., Zhang, J. and Yu, M. 2016b. Uniform design ray in the assessment of combined toxicities of multi-component mixtures. Sci. Bull., 61: 52-58.

Luo, Y.H., Lai Y.J.S., Zheng C.W., Iihan, Z, E., Ontiveros V.A., Long, X.X., Krajmalnik, B.R. and Rittmann B.E. 2020. Increased expression of antibiotic-resistance genes in biofilm communities upon exposure to cetyltrimethylammonium bromide (CTAB) and other stress conditions. Sci. Total Environ., 765: 144-164.
Mo, L.Y., Ma, W., Kong, S., Qin, L.T., Liang, Y.P. and Dai, J.F. 2020a. Study of the Binding Mode of Quaternary Ammonium Cationic Surfactant to Firefly Luciferase and the Prediction of Binary Mixture Toxicity. Chinese J. Struct. Chem., 39(6): 1167-1177.

Mo, L.Y,, Liu, Y.A., Zhu, J., Qin, L.T., Liang, Y. P. and Zeng, H.H. 2020b. Benefits from hazards benefit from nothing, and benefits from benefits: the combined effects of five quaternary ammonium compounds to Vibrio qinghaiensis Q67. Environ. Sci. Europe, 32(1): 35.

OECD. 2004. The 2004 OECD List of High Production Volume Chemicals. http://www.oecd.org/ chemical safety/ risk-assessment/33883530. pdf. (Accessed 5 Sept. 2004).

Oh, S., Kurt, Z., Tsementzi, D., Weigand, M.R., Kim, M., Hatt, J.K., Tandukar, M., Pavlostathis, S.G., Spain, J.C. and Konstantinidis, K.T. 2014. Microbial community degradation of widely used quaternary ammonium disinfectants. Appl. Environ. Microbiol., 80: 5892-5900.

Olkowska, E., Polkowska, Z. and Namie-Snik, J. 2013. A solid-phase extraction ion chromatography with conductivity detection procedure for determining cationic surfactants in surface water samples. Talanta, 116: 210-216.

Ostman, M., Lindberg, R.H., Fick, J., Bjorn, E. and Tysklind, M. 2017. Screening of biocides, metals, and antibiotics in Swedish sewage sludge and wastewater. Water Res., 115: 318-328.

Perez, E. and Hoang, T.C. 2017. Chronic toxicity of binary-metal mixtures of cadmium and zinc to Daphnia magna. Environ. Toxicol. Chem., 36: $2739-2749$.

Qu, R., Xiao, K., Hu, J., Liang, S., Hou, H. J., Liu, B.C., Chen, F., Xu, Q., $\mathrm{Wu}, \mathrm{X}$. and Yang, J. K. 2019. Predicting the hormesis and toxicological interaction of mixtures by an improved inverse distance weighted interpolation. Environ. Int., 130: 104892-104900.

Rosety, M., Ordonez, F. and Rosety, R.M. 2001. Acute toxicity of anionic surfactants sodium dodecyl sulfate (SDS) and linear alkylbenzene sulphonate (LAS) on the fertilizing capability of gilthead (Sparus aurata L) sperm. Histol. Histopathol., 16(3): 839-843.

Ruan, T.S., Song, S., Wang, T., Liu, R., Lin, Y. and Jiang, G. 2014. Identification and composition of emerging quaternary ammonium compounds in municipal sewage sludge in China. Environ. Sci. Technol., 48(8): 4289-4297.

Sprague, J.B. and Ramsay, B.A. 1965. Lethal levels of mixed copper-zinc solution for juvenile salmon. J. Fish. Res. Board Can., 22: 425-432.

Vannini, A., Paoli, L., Vichi, M., Bačkor, M., Bačkorová, M. and Loppi, S. 2018. Toxicity of diclofenac in the fern azolla filiculoides and the lichen xanthoria parietina. Bull. Environ. Contam. Toxicol., 100: 430-437.

Warne, M. and Hawker, D. 1995. The number of components in a mixture determines whether synergistic and antagonistic or additive toxicity predominate: The funnel hypothesis. Ecotoxicol Environ. Safety, 31(1), 23-28.

Xu, Y.Q., Liu, S.S., Fan, Y. and Li K. 2018. Toxicological interaction of multi-component mixtures to Vibrio qinghaiensis sp.-Q67 induced by at least three components. Sci. Total Environ., 635: 432-442.

Yu, M., Liu, S.S., Wang, M.C., Chen, F. and Tang, H. 2014. Mixture toxicities of three pesticides having different time-toxicity profiles. Chinese J. Chem., 32: 545-552.

Yuan, J., Liu, S.S., Wang, L.J. and Shao, Y.M. 2011. Optimization of microplate toxicity analysis method based on Chlorella Pyrenoidose. Res. Environ. Sci., 24: 553-558.

Zhang, C., Cui, F., Zeng, G.M., Jiang, M., Yang, Z.Z., Yu, Z.G., Zhu, M.Y. and Shen, L.Q. 2015. Quaternary ammonium compounds (QACs): A review on occurrence, fate, and toxicity in the environment. Sci. Total Environ., 518-519: 352-362. 